\title{
RELAÇÃO ENTRE VARIÁVEIS CLIMÁTICAS E MORTALIDADE POR DOENÇAS DO APARELHO CIRCULATÓRIO EM IDOSOS NO MUNICÍPIO DE PARANAVAÍ - PR
}

\author{
RELATIONSHIP BETWEEN CLIMATE VARIABLES AND MORTALITY FOR DISEASES \\ OF CIRCULATORY APPARATUS IN OLD PEOPLE IN THE CITY OF PARANAVAÍ - PR
}

\author{
VINICIUS FERNANDES'; MAYSA DE LIMA LEITE²
}

1-Acadêmico de Medicina da Universidade Estadual de Ponta Grossa (UEPG) (Ponta Grossa, PR, Brasil). 2 -Professora da Universidade Estadual de Ponta Grossa (UEPG) (Ponta Grossa, PR, Brasil).

Autor para correspondência: viniciusfbio@hotmail.com

\section{RESUMO:}

A mortalidade por doenças do aparelho circulatório e sua relação com o meio ambiente têm sido alvo de estudos ao longo dos anos. Sendo assim, o clima pode influenciar negativamente a saúde dos indivíduos de determinadas populações mais vulneráveis como os idosos. Realizou-se o presente trabalho com o objetivo de analisar a relação das variáveis climáticas sobre as doenças do sistema circulatório associado aos idosos de um município do Paraná. Trata-se de um estudo transversal realizado entre os anos 1998 a 2014 contemplando idosos do município de Paranavaí-PR com dados coletados a partir do Departamento de Informática do Sistema Único de Saúde (DATASUS). Comparou-se a taxa de mortalidade junto às variáveis climáticas: temperaturas médias e pluviosidade obtidos do Instituto Agronômico do Paraná (IAPAR). Efetuou-se para tanto, tabulação dos dados e confecção de gráficos para interpretação dos mesmos. As taxas de mortalidade a despeito das doenças cerebrovasculares se mostraram inversamente proporcionais às temperaturas médias e à pluviosidade em sua maioria, relação que se manteve semelhante para as demais doenças (isquêmicas, hipertensivas e outras). Além disso, a idade elevada firmouse como um pilar para os efeitos deletérios do meio ambiente sobre a saúde dessa população que por vezes se mostra mais vulnerável com o decorrer dos anos. Portanto, as associações demonstradas no estudo denotam a fragilidade do grupo de idosos da cidade em específico em relação às condições climáticas estudadas.

Palavras-chave: Doenças cardiovasculares, sazonalidade, mortalidade

\section{ABSTRACT:}

The mortality due to diseases of the circulatory system and their relationship with the environment, with the objective of studies over the years. Therefore, the climate can negatively influence the health of the user of certain vulnerable populations such as the elderly. The present work was performed with the objective of analyze the relationship of climatic variables on diseases of the circulatory system associated with the elderly in a municipality of Paraná. It is a cross-sectional study among the years 1998 to 2014 included the elderly of the municipality of Paranavaí-PR with data collected from the Departamento de Informática do Sistema Único de Saúde (DATASUS). Mortality rate was then compared to the climatic variables: average temperatures and rainfall, obtained from the Instituto Agronômico do Paraná (IAPAR). Data were tabulated and graphs were created for the interpretation of the data. The mortality rates in spite of cerebrovascular diseases are inversely proportional to average temperatures and rainfall to their own majority, which are maintained for other diseases (ischemic, hypertensive and more). In addition, high age has 
established itself as a pillar for the deleterious effects of the environment on the health of this population which is sometimes more vulnerable over the years. Therefore, the associations demonstrated in the study denote the fragility of the elderly group of the city in specific in relation to the climatic conditions studied.

Keywords: Cardiovascular diseases, seasonality, mortality

\section{INTRODUÇÃO}

O notório advento do aumento na expectativa de vida da população em diversos países nos últimos anos gerou uma concentração de mortalidade num período em que as pessoas se tornaram idosas permitindo que mais de $60 \%$ das mortes se concentrassem nesse período. As melhores condições de vida e saúde a essa população específica se apresenta como um desafio aos serviços de saúde, visto que há uma mudança lenta e evolutiva no perfil das patologias que determinam a mortalidade para os dias atuais. A identificação desse perfil representa um maior entendimento do que poderia ser feito em relação às manobras intervencionais relacionadas ao tema (CABRERA, M.A.S.; ANDRADE, S.F.; WAJNGARTEN, M. 2007, p.12-8).

O aparelho circulatório pode ser visto como um exemplo de sensibilidade humana; composto de um sistema vascular complexo, ele exerce basicamente a função de garantir a correta circulação sanguínea a diversos órgãos e tecidos do corpo humano, trabalhando de forma eficiente a uma temperatura média de $37^{\circ} \mathrm{C}$. Esse processo pode ser desregulado por variações do clima e tempo que acionam processos homeostáticos de adequação sistêmicas do corpo como constrição ou dilatação dos vasos sanguíneos que por vezes a depender do estado de saúde do indivíduo, dentre outros fatores, podem levar a aumento da carga de pressão, elevando o risco de doenças circulatórias (TORTORA, 2016, p. 394). Dito isso, há tempos, estudos vem correlacionando a influência do meio ambiente sobre a humanidade, tentando compreender esse processo complexo de saúde/doença, resultante de ação da sociedade em face da apropriação da natureza e organização do espaço (MURARA et al. 2010, p. 54).

Segundo Moraes et al. (2009, p. 638), as doenças do aparelho circulatório podem ser vistas como uma das principais causas de óbitos em países desenvolvidos e em desenvolvimento. Em adição, Mansur (2012, p. 757) corrobora esses dados trazendo que as patologias relacionadas ao sistema cardiovascular representam a primeira causa de morte no Brasil, sendo que são baixos os números de estudos que abordam a distribuição temporal e espacial da mortalidade por elas, sobretudo quando se trata de idosos. Seus diferentes fatores de riscos estão relacionados desde comportamentais até genéticos e por 
esse motivo a distribuição territorial e o processo de urbanização tem íntima relação com a forma com que esses grupos selecionados lidam com esse conjunto de doenças.

Sendo assim, é nítido que dentre os fatores que levam ao agravo e à mortalidade por essas doenças, alguns fatores climáticos estejam intimamente relacionados. O clima e os diferentes tipos de tempo (ondas de calor, períodos de estiagem, variações súbitas das temperaturas) são entendidos como "um fator ambiental que influencia o organismo humano, não com um caráter determinista, mas como um elemento que pode contribuir de maneira benéfica ou maléfica para a saúde humana" (MURARA et al. 2010, p. 55).

Nesse sentido, pode-se afirmar que a incidência de determinadas doenças está relacionada diretamente com a tropicalidade de algumas regiões do planeta. Essas doenças tendem a ser predominantes em certas zonas climáticas por, exatamente, oferecerem condições bastante favoráveis para a proliferação dos vetores responsáveis pela sua transmissão (ARAÚJO \& NUNES, 2005, p. 101).

O objetivo do presente estudo consiste em verificar a relação que as variáveis climáticas, em especial temperatura média e precipitação pluviométrica, exercem sobre o sistema circulatório considerando-se uma perspectiva saúde/doença num determinado espaço geográfico aliado ao avançar da idade de uma população específica.

\section{MATERIAL E MÉTODOS}

Tratou-se de um estudo transversal, cuja unidade de análise de área foi o município de Paranavaí situado no estado do Paraná. Os dados de mortalidade considerados no estudo contemplam o período de janeiro de 1998 a dezembro de 2014. A figura abaixo demonstra a cidade do estudo em destaque.

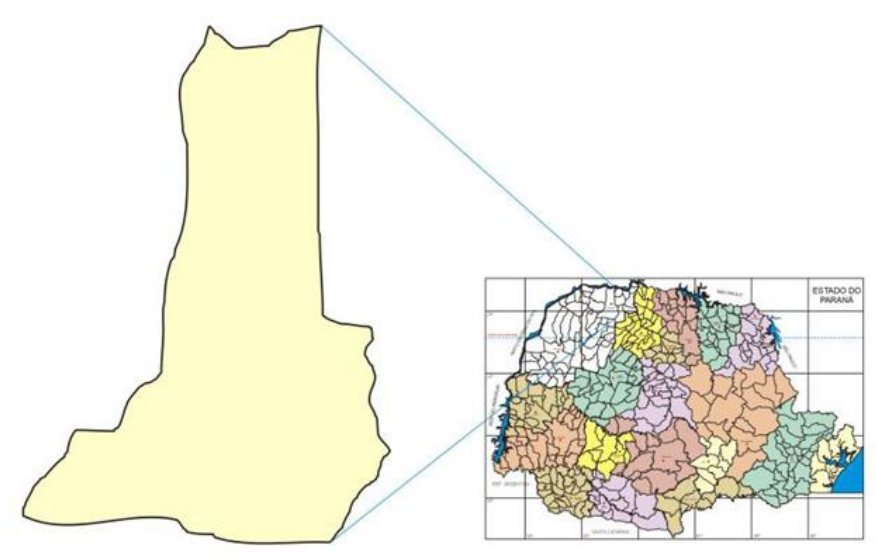

Figura 1 - Mapa do Paraná - Em destaque a cidade de Paranavaí-PR. Fonte: Governo do Estado do Paraná 
A população estudada consistiu de idosos residentes no município citado anteriormente que foram a óbito por doenças do aparelho circulatório como cerebrovasculares, doenças isquêmicas do coração e hipertensão arterial sistêmica. Para inclusão no estudo, considerou-se a faixa etária maior que 60 anos onde a mesma foi dividida em 3 grupos principais: entre 60 e 69 anos, de 70 a 79 anos e maiores de 80 anos.

Os dados foram obtidos do Departamento de Informática do Sistema Único de Saúde (DATASUS), no site do Ministério da Saúde (http://datasus.saude.gov.br). Esse sistema segue uma padronização de classificação estatística internacional de doenças e problemas relacionados à saúde (CID 10). As doenças do aparelho circulatório correspondentes ao capítulo IX. Para isso, as informações coletadas foram número de óbitos e faixa etária correspondente às idades já mencionadas.

Assim sendo, essas informações podem ser acessadas via internet para consulta livre na forma de dados agregados por municípios, ou seja, as mesmas não foram coletadas de maneira individualizada e nominal, diminuindo assim qualquer possibilidade de dano de ordem física ou moral na perspectiva do indivíduo e das coletividades.

Optou-se por realizar, simultaneamente, a comparação das taxas de mortalidade (equação 1) junto às variáveis climáticas, onde seus dados históricos de temperatura média e pluviosidade foram obtidos junto ao Instituto Agronômico do Paraná (IAPAR), (235' S latitude, $52^{\circ} 26^{\prime}$ W longitude e $480 \mathrm{~m}$ altitude). Para a complementação da série foi utilizado o Gerador Climático PGCLIMA_R.

Equação 1: $C M=\left(\frac{\text { Número de óbitos de idosos por DAC }}{\text { População de idosos na metade do período }}\right) * 10.000$

Figura 2: Equação para comparação de mortalidade.

Na equação 1 acima, para o cálculo do Coeficiente de Mortalidade utilizou-se a média da população idosa central (junho e julho) de cada ano. Sendo assim, o número de casos de cada mês foi dividido pela média da população central do seu respectivo ano, e na sequência, multiplicado por 10.000 habitantes.

De posse dos dados efetuou-se a tabulação e triagem utilizando-se as planilhas eletrônicas do EXCEL®, as quais permitiram a elaboração de gráficos de distribuição mensal, sazonal e anual, possibilitando a análise e identificação das ocorrências das doenças. 
Neste estudo, inicialmente efetuou-se a análise descritiva das variáveis utilizadas e na sequência realizou-se a análise de inferência estatística associada aos principais índices epidemiológicos pertinentes.

\section{RESULTADOS E DISCUSSÃO}

Os gráficos construídos a seguir têm por objetivo demonstrar a relação entre mortalidade por determinadas doenças relacionadas ao aparelho circulatório, a sazonalidade e a precipitação pluviométrica num período pré-estipulado de forma a simplificar o entendimento dessas possíveis interferências. Para tanto, estratificou-se a mortalidade por grupos de faixas etárias para assim, melhor entender o comportamento de cada um numa determinada patologia.

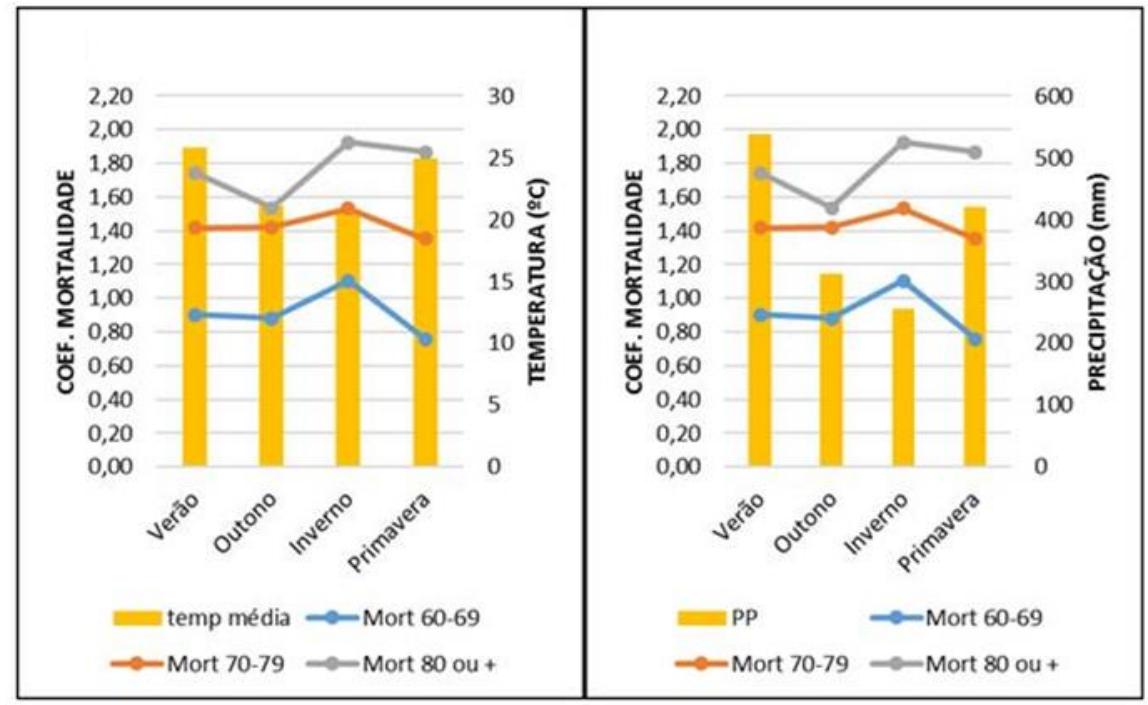

Figura 3: Doença cerebrovascular - relação entre doença, mortalidade, temperatura média e precipitação pluviométrica.

Observa-se na Figura 3 um aumento na mortalidade relacionada às doenças cerebrovasculares quando se diminuem as temperaturas médias do período em todas as faixas etárias estudadas. O contrário também se apresentou verdadeiro ocorrendo, portanto, um decréscimo na mortalidade quando as temperaturas começaram a se elevar. Percebe-se ainda que a faixa etária onde a mortalidade teve maior impacto foi do grupo de idosos maiores de 80 anos, demonstrando assim os prejuízos para a saúde principalmente dos mais experientes. Relação semelhante ocorre quando se observa em períodos onde 
há uma menor precipitação, o que coincide com o período de menores temperaturas médias, ou seja, intervalos frios e secos há maior taxa de mortalidade envolvida.

McMichael et al. (2008, p. 1127) corrobora os estudos do autor citado acima, demonstrando o papel do calor para com os índices de mortalidade por patologias cardiovasculares em cidades com poder aquisitivo menor como Salvador, São Paulo, Santiago, Cidade do Cabo entre outras. Mas não somente, pois o frio em cidades como Cidade do México e Bangkok também apresentam padrões de mortes conexas a baixas temperaturas. Esses padrões contemplam tanto fatores climáticos como não climáticos, como poluição do ar, condições sanitárias e de economia.

Já somente em relação ao IAM associado à temperatura média num determinado período, Sharovsky (2001, p. 04) demonstrou que os 12.007 casos por IAM no município de São Paulo estavam diretamente e inversamente relacionados às altas e baixas temperaturas respectivamente. Mostrou-se então menor mortalidade entre $21,6^{\circ} \mathrm{C}$ e $22,6^{\circ} \mathrm{C}$, ocorrendo um aumento abaixo e acima desta faixa, o que sugere um efeito deletério dos extremos de temperatura.

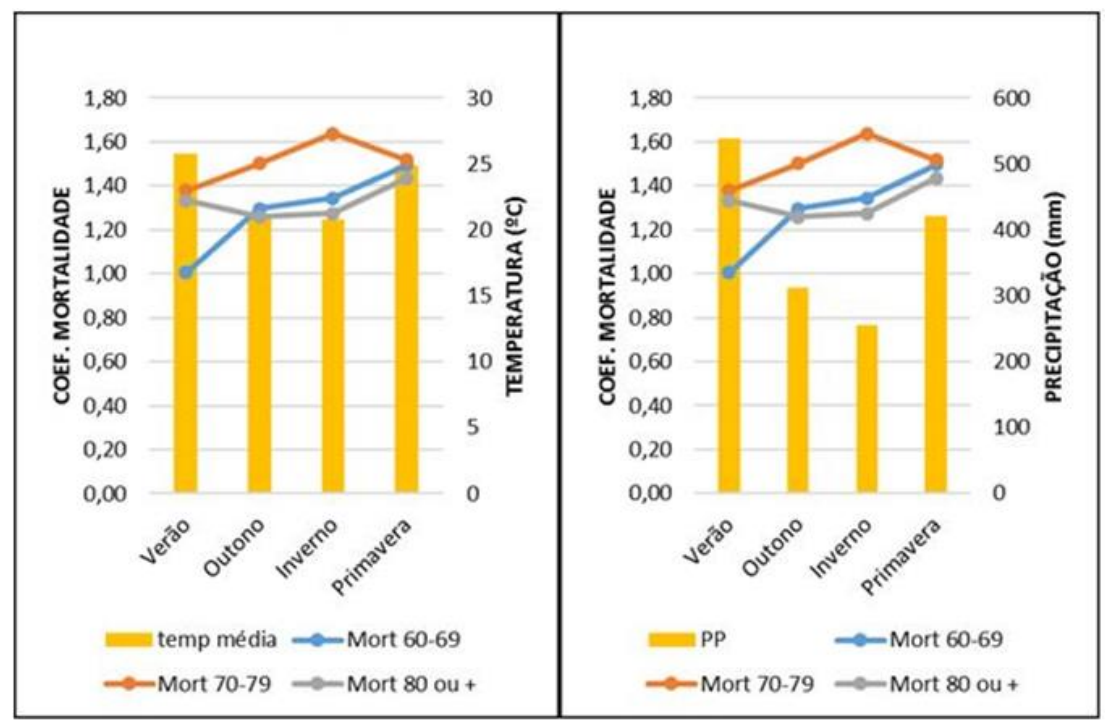

Figura 4: Doenças Isquêmicas - relação entre doença, mortalidade, temperatura média e precipitação pluviométrica.

Quanto às doenças isquêmicas, nota-se na Figura 4 um padrão semelhante ao já descrito para as doenças cerebrovasculares, onde nos meses com temperaturas médias menores, representados pelas estações outono e inverno, há um aumento na mortalidade. Mas diferentemente dos primeiros gráficos, o número de idosos que morreram entre 70 e 
79 anos por doenças isquêmicas foram maiores aqui, seguidos pela faixa etária dos 60 aos 69 anos e por último pelos maiores de 80 anos. Situação semelhante ocorre em comparação à precipitação pluviométrica, apesar da variável climática ser diferente.

Contrapondo Sharovsky (2001, p. 04), os estudos de Braun (2003, p. 65) que relacionam sazonalidade com doenças do aparelho cardiovascular mostram significância de mortalidade com evidente aumento do número de óbitos no inverno e queda durante o verão entre os anos 1996 a 2000. Além desse, outros estudos mostraram essa mesma relação como Pitton e Domingos (2004), Castilho (2006, p. 15), Pascoalino (2008) e Mitchell, Blane e Bartley (2002, p. 836-37), constatando em comum uma tendência sazonal do desenrolar das patologias circulatórias, sendo, portanto, de maior ocorrência nos períodos de baixas temperaturas e umidade, especialmente em julho e agosto.

Ainda, observou-se no estudo de Analitis et al. (2008, p. 1403-04), que quando houve diminuição de $1^{\circ} \mathrm{C}$ na temperatura obteve-se aumento da mortalidade diária de $1,72 \%$ com intervalo de confiança de $95 \%(1,44 ; 2,01)$ relacionados a doenças cardiovasculares e $1,25 \%$, também com intervalo de confiança de $95 \%(0,77 ; 1,73)$ quando associados a doenças cerebrovasculares. Nota-se, portanto, que as temperaturas mais baixas são efetivamente danosas à saúde e que isso não pode ser banalizado, visto que as mudanças climáticas globais têm se mostrado presentes de forma impactante ao longo dos anos (DA SILVA, et al. 2015, p. 03-04). Em adição, o estudo do Eurowinter Group (1997, p. 1343) mostrou que essa variação da temperatura também está relacionada a mortes por doenças isquêmicas.

Em consonância, os estudos de Vasconcelos et al (2013, p. 15-16) também demonstraram a relevância das temperaturas mais baixas para doenças cardiovasculares em Portugal, onde essa relação não estava muito bem estabelecida. Foi a primeira vez que o infarto agudo do miocárdio foi avaliado e sua relação com o impacto que o frio traz à saúde da população melhor estabelecida. Verificou-se com isso que tanto a população geral estudada, quanto os idosos maiores de 65 anos obtiveram maiores taxas de internações se comparados aos períodos mais quentes, assim, consequentemente, elevam-se os riscos desses indivíduos chegarem a óbito pela doença ou por suas complicações.

Dilaveris, et al. (2006, p. 1748) analisaram as consequências e a força das variáveis climáticas em relação à mortalidade por doenças do coração, como o infarto agudo do miocárdio (IAM) em 2001, fazendo uso de dados diários de temperatura, pressão atmosférica e umidade relativa do ar. Obtiveram 3126 casos, sendo que a média diária de 
mortes fora aproximadamente $32 \%$ maior no período estudado com temperaturas mais baixas (inverno) em relação ao verão, especialmente na população mais idosa. Apesar disso, o mês de dezembro contempla mais de 2 vezes o número de mortes que o período que compreende junho, podendo ser explicado pelo maior grau de instrução que esses idosos têm no período relatado sobre os perigos e as consequências do frio para a sua saúde.

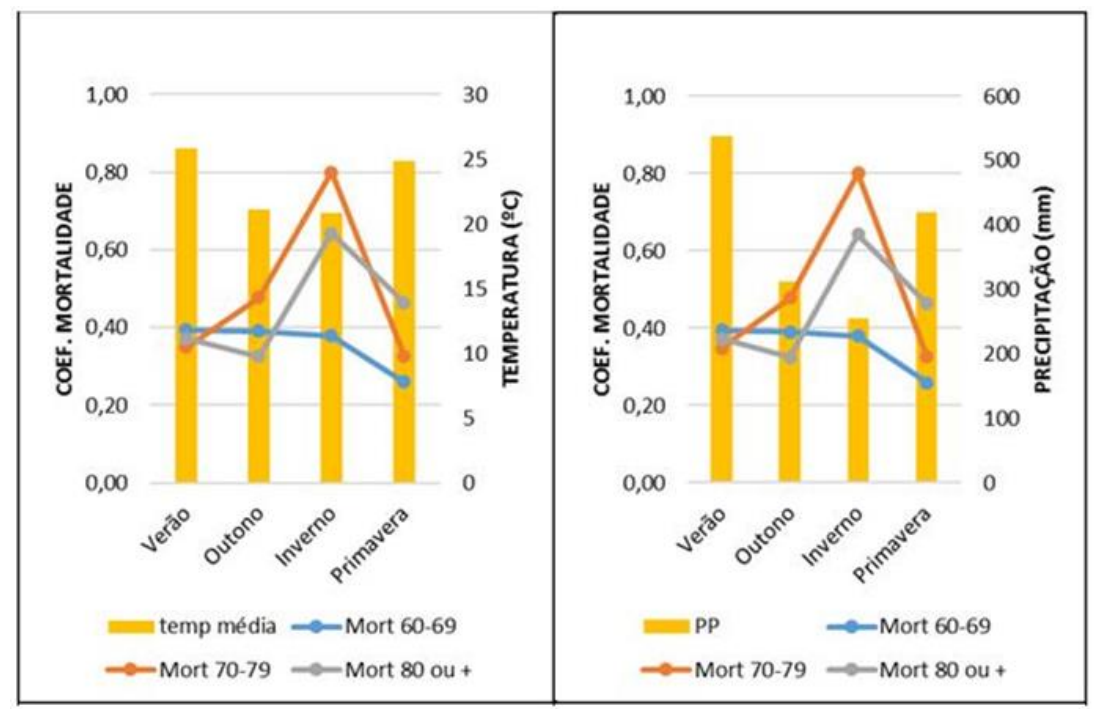

Figura 5: Doença Hipertensiva - relação entre doença, mortalidade, temperatura média e precipitação pluviométrica.

Quando se analisa a doença hipertensiva e a confronta com a temperatura média, nota-se um comprometimento semelhante aos gráficos anteriores, onde de maneira geral verifica-se uma ascensão da mortalidade quando as temperaturas médias do ar estão mais baixas (figura 5). No entanto, a faixa etária onde se mostra com maior incidência de mortalidade está entre os 70 e 79 anos, seguido pelos mais idosos (80 anos ou mais) e por último a população entre 60 e 69 anos que se mostra praticamente estável e até queda mínima durante o inverno e queda mais significativa quando a temperatura começa a se elevar. Quanto à precipitação, a figura demonstra um comportamento semelhante, ou seja, picos de mortalidade nos meses de outono e inverno, onde a precipitação se mantém reduzida e diminuição da mortalidade nos meses mais quentes.

Quando se abordam doenças cardiovasculares na atualidade, têm-se autores como Muller (2015, p.14) trazendo evidências de elevação da mortalidade tanto em homens quanto em mulheres concomitantes à idade, sendo que os riscos são sempre maiores nos 
homens independente do grupo etário do estudo. Isso pode ser explicado por acessarem menos os serviços de saúde e, por conseguinte cuidarem-se menos. Mas não somente, no estudo de Malta e colaboradores (2017 p.164) foi demonstrado que ao longo de 2006 a 2014 os homens apresentaram maior prevalência de tabagismo que as mulheres, configurando, portanto, um importante fator de risco para eventos cardiovasculares. No entanto, evidencia-se por outro lado um declínio global na mortalidade pelo grupo dessas doenças, em especial no estado do Paraná, onde uma explicação plausível pode estar na abrangência do acesso à saúde através da atenção básica, além da tecnologia como recurso para diagnósticos e tratamentos.

Corroborando o estudo anterior, em alguns países desenvolvidos têm-se observado um declínio na tendência de mortalidade por doenças do aparelho circulatório a partir do final da década de 60. Com isso, na década de 1980, houve um comportamento de decréscimo em torno de 3,5\% ao ano (MCGOVERN, 1996, p. 884; FORD, 2007, p. 2129). Já em nosso país, essa diminuição só começou a ser observada em 1970. Apesar de vários estudos confirmarem esse declínio em algumas regiões do Brasil, as hipóteses que tentam explicar tal fenômeno são provindas de resultados de estudos internacionais, não se podendo, portanto, afirmar quais os determinantes dessa queda. Algumas hipóteses são criadas para tentar explicar, como maior acesso à assistência médica, melhorias no diagnóstico e compreensão da fisiopatologia dessas doenças (MORAES, et al. 2009, p.638).

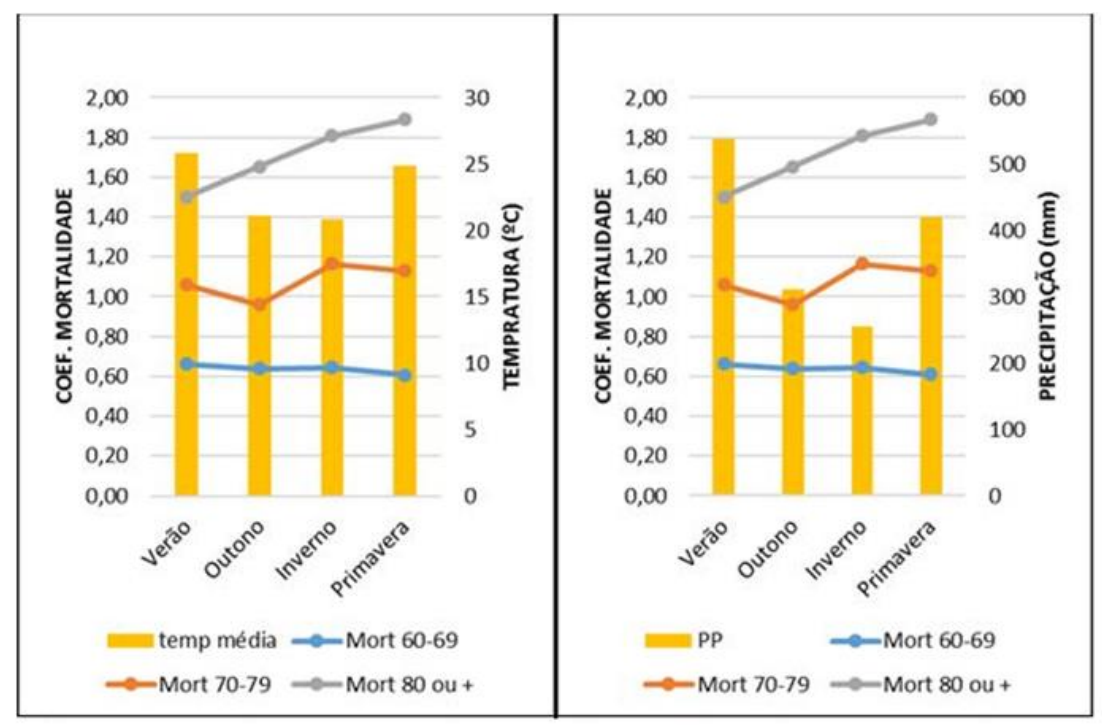

Figura 6: Outras Causas - relação entre doença, mortalidade, temperatura média e precipitação pluviométrica. 
Outras causas, demonstradas pela figura 6 , englobam inúmeras doenças que não encenam dentre as principais causas de morte, mas ainda assim tem seu valor confirmado, como por exemplo, insuficiência cardíaca, embolia e trombose arteriais, dissecção de aorta, dentre outros. A tendência da figura 5a mostra que o grupo mais idoso apresenta picos de mortalidade passando tanto pelos meses com temperaturas menos elevadas quanto à primavera com meses mais quentes. Em seguida nota-se que a população compreendida entre 70 e 79 anos figura com um pico de mortalidade no inverno e o último grupo, dos 60 aos 69, varia bem pouco sua dinâmica. Ao analisar-se o coeficiente de mortalidade associado à precipitação pluviométrica observa-se a mesma tendência já descrita para temperatura média.

Existe grande relação entre a termorregulação, a regulação circulatória e o meio físico que se mostram mais típicas em zonas urbanas. Constata-se assim que situações de estresse onde o organismo precisa se adaptar a uma determinada queda ou aumento de temperatura, produzem uma sobrecarga da termorregulação comprometendo a saúde dos indivíduos. Para isso diversos estudos pelo mundo analisaram patologias do sistema circulatório mostrando que pessoas são realmente comprometidas pelas variações climáticas, como por exemplo, as temperaturas do ambiente (DA SILVA, et al. 2015, p. 08).

Sendo assim, as ondas de frio e de calor que colocam os idosos frente a situações para as quais eles não estão devidamente adaptados devido às deleções que a idade traz com os anos vividos, extrapolam suas capacidades de regulação térmica e quando exigidos demais a doença que já se instalou se agrava e pode levá-los à morte (FOGAÇA \& LIMBERGER, 2015, p. 139). Da Silva (2015, p. 08) também revela que os trabalhos que se focalizam em ondas de calor e frio demonstram as associações com os problemas de saúde, sobretudo nas comunidades mais vulneráveis como os idosos (já citados), além de crianças e dos que vivem em situações de pobreza.

Em termos de saúde, podemos considerar a existência de uma relação direta com o ambiente. O clima, por exemplo, tem mudanças contínuas e danosas que afetam o meio social do indivíduo, assim, favorecendo o aparecimento de determinados agravos à saúde. Alguns elementos como temperatura do ar, precipitação, umidade, pressão atmosférica, ventos etc., interferem no bem-estar das pessoas, mas não somente, pois quando são associados a caracteres tanto físicos quanto psicológicos, culturais, e fatores de risco ligados ao estilo de vida como dieta e obesidade, exercícios físicos, tabagismo, níveis de colesterol, fatores de coagulação e suscetibilidade contribuem para o agravamento de determinadas doenças (MURARA et al. 2010, p. 55). 


\section{CONSIDERAÇÕES FINAIS}

Portanto, demonstrou-se que as variáveis climáticas temperatura média do ar e precipitação pluviométrica no município de Paranavaí, estado do Paraná, puderam influenciar efetivamente nas taxas de mortalidade por doenças do aparelho circulatório, visto que ao se comparar os meses mais frios e menos chuvosos aos mais quentes e mais chuvosos, o número de idosos que vieram a óbito nos primeiros é claramente mais evidente.

Observou-se ainda que a incidência de óbitos segundo a faixa etária se mostrou dinâmica, pois nem sempre os mais velhos eram os que mais morriam devido a uma determinada enfermidade do grupo das doenças do aparelho circulatório. Assim, apesar do notório entendimento de que indivíduos mais experientes sofram mais por essas patologias devido a influências climáticas, é evidente a necessidade de estudos que abordem outras relações a fim de se evitar generalizações a respeito do tema.

Por conseguinte, faz-se possível inferir a importância da relação existente entre determinantes climáticos e o processo saúde-doença, visto que a população específica de idosos estudada se mostra em situação vulnerável devido a diversos fatores, sendo eles ambientais e não ambientais. Esse processo pode, claramente, ser agravado pelas intempéries e condições de vida de cada indivíduo, sobretudo quando esses personagens se mostram em situações de carência e falta de informação. Portanto, após a exposição dos resultados, percebe-se a necessidade de mais estudos que contemplem a complexidade da população idosa e suas particularidades a fim de correlacionar outras relações existentes entre os fatores relacionados ao clima e as doenças do aparelho circulatório.

Deste modo, as relações existentes entre as características climáticas e a saúde, principalmente dos idosos, servem de base para a elaboração de políticas públicas que visem a qualidade de vida desse grupo, sendo, portanto, peça chave na advertência pelos serviços de meteorologia para prevenção das patologias mais presentes.

\section{REFERÊNCIAS}

ANALITIS, Antonis et al. Effects of Cold Weather on Mortality: Results From 15 European Cities Within the PHEWE Project. American Journal of Epidemiology, 2008, vol. 168, oㅜ 12, p. 1397-1408. 
ARAÚJO, Ronaldo Rodrigues; NUNES, José Sérgio Alves. Relações geográficas entre o clima e a incidência de dengue na cidade de São Luís-MA. Ciências Humanas em Revista, São Luís, v. 3, n. 2, p. 93-108, 2005.

BRAUN, Samuel. Influências Meteorotrópicas nas Doenças Cardiovasculares na Cidade de São Paulo. 2003. Dissertação de Mestrado. Universidade de São Paulo, $148 f$.

CABRERA, Marcos Aparecido Sarria; ANDRADE, S. M.; WAJNGARTEN, Maurício. Causas de mortalidade em idosos: estudo de seguimento de nove anos. Revista Brasileira de Geriatria e Gerontologia, v. 1, n. 1, p. 12-8, 2007.

CASTILHO, F. J. V. Abordagem Geográfica do Clima Urbano e das Enfermidades em São José do Rio Preto/SP. Dissertação de mestrado, UNESP-IGCE. Rio Claro (SP), 2006, $228 f$.

DA SILVA, Edelci Nunes; RIBEIRO, Helena; SANTANA, Paula. Clima e saúde em contextos urbanos: uma revisão da literatura. Revista Bibliográfica de Geografía y Ciencias Sociales, Barcelona: Universidad de Barcelona, v. 19, n. 1092, 2014.

DILAVERIS, P. et al. CLimate Impacts on Myocardial infarction deaths in the Athens TErritory: the CLIMATE study. Heart, v. 92, n. 12, p. 1747-1751, 2006.

GROUP, The Eurowinter. Cold exposure and winter mortality from ischaemic heart disease, cerebrovascular disease, respiratory disease, and all causes in warm and cold regions of Europe. The Lancet, v. 349, n. 9062, p. 1341-1346, 1997.

FOGAÇA, Thiago Kich; LIMBERGER, Leila. PERCEPÇÃO AMBIENTAL E CLIMÁTICA: ESTUDO DE CASO EM COLÉGIOS PÚBLICOS DO MUNICÍPIO DE TOLEDO-PR. Revista do Departamento de Geografia, v. 28, p. 134-156, 2015.

FORD, Earl S.; CAPEWELL, Simon. Coronary heart disease mortality among young adults in the US from 1980 through 2002. Journal of the American College of Cardiology, v. 50, n. 22, p. 2128-2132, 2007.

MANSUR, Antonio de Padua; FAVARATO, Desidério. Mortalidade por doenças 
cardiovasculares no Brasil e na região metropolitana de São Paulo: atualização 2011. Arquivo brasileiro de cardiologia, v. 99, n. 2, p. 755-761, 2012.

MCGOVERN, Paul G. et al. Recent trends in acute coronary heart disease mortality, morbidity, medical care, and risk factors. New England Journal of Medicine, v. 334, n. 14, p. 884-890, 1996.

MCMICHAEL, Anthony J. et al. International study of temperature, heat and urban mortality: the 'ISOTHURM' project. International journal of epidemiology, v. 37, n. 5, p. 1121-1131, 2008.

MITCHELL, R.; BLANE, D.; BARTLEY, M. Elevated risk of high blood pressure: climate and the inverse housing law. International Journal of Epidemiology, Great Britain, n. 31, p. 831 $-838,2002$.

MORAES, Suzana Alves de et al. Mortalidade por doenças do aparelho circulatório no município de Ribeirão Preto-SP, de 1980 a 2004. Arquivo Brasileiro de Cardiologia, v. 93, n. 6, p. 637-44, 2009.

MULLER, Erildo Vicente; GIMENO, Agostinho; GODOY, Suely. Mortalidade por doenças cardiovasculares segundo gênero e idade no Estado do Paraná, Brasil: 1979 a 1981 e 2006 a 2008. Cadernos Saúde Coletiva, v. 23, n. 1, 2015.

MURARA, Pedro Germano; AMORIM, Margarete Cristiane de Costa Trindade. Clima e saúde: variações atmosféricas e óbitos por doenças circulatórias. Revista Brasileira de Climatologia, v. 6, p.79-92, 2010.

MURARA, Pedro Germano; COELHO, M. S. Z. S.; AMORIM, M. C. C. T. Análise da influência meteorológica nas internações por doenças cardiovasculares. Caderno Prudentino de Geografia, v. 32, n. 1, p. 53-65, 2010.

PASCOALINO, A. Condições Climáticas e suas Implicações nas Crises Hipertensivas dos Citadinos de Limeira - SP. Simpósio de Pós Graduação em Geografia do Estado de São Paulo, 2008. 
PITTON, S. e DOMINGOS, A. Tempo e Doenças: Efeitos dos Parâmetros Climáticos nas Crises Hipertensivas nos Moradores de Santa Gertrudes - SP. Estudos Geográficos: Revista Eletrônica de Geografia, ESTGEO v.2, n.1, 2004.

RUMEL, Davi et al. Infarto do miocárdio e acidente vascular cerebral associados à alta temperatura e monóxido de carbono em área metropolitana do sudeste do Brasil. Revista de saúde pública, v. 27, n. 1, p. 15-22, 1993.

SHAROVSKY, R. Efeitos da temperatura e poluição do ar na mortalidade por infarto agudo do miocárdio no município de São Paulo. Tese de Doutorado. Tese (Doutorado em Cardiologia) - Faculdade de Medicina da Universidade de São Paulo, 2001, 86f.

TORTORA, Gerard J.; DERRICKSON, Bryan. Corpo Humano-: Fundamentos de Anatomia e Fisiologia. Porto Alegre: Artmed Editora, 2016, 704p.

VASCONCELOS, João et al. The impact of winter cold weather on acute myocardial infarctions in Portugal. Environmental pollution, v. 183, p. 14-18, 2013. 